\title{
Damage Detection of Structures for Ambient Loading Based on Cross Correlation Function Amplitude and SVM
}

\author{
Lin-sheng Huo, ${ }^{1} \mathrm{Xu} \mathrm{Li},{ }^{1}$ Yeong-Bin Yang, ${ }^{2}$ and Hong-Nan $\mathrm{Li}^{1}$ \\ ${ }^{1}$ Key Laboratory of Coastal and Offshore Engineering, Dalian University of Technology, Dalian 116024, China \\ ${ }^{2}$ Department of Civil Engineering, National Taiwan University, Taipei, Taiwan \\ Correspondence should be addressed to Lin-sheng Huo; lshuo@dlut.edu.cn
}

Received 17 November 2015; Accepted 1 March 2016

Academic Editor: Abdollah Shafieezadeh

Copyright (c) 2016 Lin-sheng Huo et al. This is an open access article distributed under the Creative Commons Attribution License, which permits unrestricted use, distribution, and reproduction in any medium, provided the original work is properly cited.

\begin{abstract}
An effective method for the damage detection of skeletal structures which combines the cross correlation function amplitude (CCFA) with the support vector machine (SVM) is presented in this paper. The proposed method consists of two stages. Firstly, the data features are extracted from the CCFA, which, calculated from dynamic responses and as a representation of the modal shapes of the structure, changes when damage occurs on the structure. The data features are then input into the SVM with the one-againstone (OAO) algorithm to classify the damage status of the structure. The simulation data of IASC-ASCE benchmark model and a vibration experiment of truss structure are adopted to verify the feasibility of proposed method. The results show that the proposed method is suitable for the damage identification of skeletal structures with the limited sensors subjected to ambient excitation. As the CCFA based data features are sensitive to damage, the proposed method demonstrates its reliability in the diagnosis of structures with damage, especially for those with minor damage. In addition, the proposed method shows better noise robustness and is more suitable for noisy environments.
\end{abstract}

\section{Introduction}

Structural damage detection is crucial in reducing catastrophic failures and prolonging the service life of structures. One of the most popular global structural damage detection techniques is the vibration-based damage detection technique, which has received considerable attention in recent years. The vibration-based damage detection methods can be classified as the model-based damage detection method (MBDDM) and non-model-based damage detection method (NMBDDM) $[1,2]$. For the model-based method, the structural model is a function of the physical properties of the structure (mass, damping, and stiffness); hence model updating techniques are needed to improve the precision of the parameters describing the structure. As most model updating techniques are complicated and their precisions are limited for complex structures, the non-model-based method, which can avoid the drawbacks of the model-based method, is considered as a better choice in general. It can be easily implemented in online Structural Health Monitoring (SHM) systems for its simple computing process.
For the NMBDDM, a precise analytical model of the structure is not required, and the damage features can be extracted from the modal parameters or dynamic responses [2]. As a huge amount of damage information can be extracted from the modal parameters, some damage features can be detected based on the changes of natural frequencies or mode shapes [3]. However, modal parameters such as the mode shapes cannot be identified precisely for complex structures, which may reduce the accuracy of the NMBDDM. Therefore, some scholars have proposed the extraction of damage features directly from the dynamic response in time domain, frequency domain, or time-frequency domain. In their researches, statistical analysis technologies including the outlier analysis [4] and independent component analysis (ICA) [5] and signal process technologies including the wavelet transform technology (WPT) $[6,7]$ and Hilbert Huang transform (HHT) $[8,9]$ have been adopted to extract damage features from the dynamic response.

Almost all of the NMBDDM mentioned above can be used only to identify the presence of damage. Yang and 
coworkers [2, 10-12] proposed a type of NMBDDM, which can be used to detect and locate damage with the correlation and relative difference between the cross correlative function amplitude vectors obtained from the intact and damaged structures. However, this method seems to have its limitation. Firstly, it is valid only for the case under steady random excitation within the specific frequency spectrum. Further, it requires the number of sensors nearly equal to that of the detectable damage locations of the structure, which means that the method may be impractical due to the high cost of the installation sensors.

It is known that the damage information provided by the damage features of the NMBDDM is generally insufficient and the locations and degree of damage are incapable of identification in full $[1,2]$. Some scholars have introduced the intelligence algorithms to the NMBDDM methods, such as the artificial neural network (ANN) $[13,14]$ for their excellent pattern recognition capability. In this connection, the damage features are used as input data, and the intelligence algorithms are introduced as the analysis tools for matching the damage patterns, detecting the damage locations, and estimating the degree of severity.

The support vector machine (SVM) is another computational method based on the statistical learning theory, of which the classification ability can be applied in damage diagnosis of structures. Compared with ANN, the SVM can be used to achieve the same global optimal solution for a smaller number of samples for its better generalization [15]. The process of damage diagnosis utilizing the SVM consists of two steps: (1) features extraction from the measured dynamic responses and (2) patterns classification based on the input vectors composed of features. The SVM allows us to recognize and classify the structural damage patterns in a way as accurate as possible. The accuracy of the SVM lies mainly in the kernel function and the damage features. Improving the kernel function such as the wavelet packet kernel function $[16,17]$ can help improve the generalization ability. The selecting of damage features should be such proper as to contain the characteristics of the structure as fully as possible. The desired damage features are sensitive to the damage and independent variables that may not be easily interfered by external factors such as excitation and noise. Previously, the data features have been extracted from the structural modal parameters [1820], independent component analysis (ICA) [21], envelope spectrum $[22,23]$, wavelet packet transform (WPT) energy spectrum [24, 25], and other statistical information [26]. Most of these data features have been proposed for the monitoring of mechanical devices, and few of them can be applied to the damage diagnosis of large and complex civil engineering structures.

The objective herein is to propose a new method that integrates the cross correlation function amplitude (CCFA) with the support vector machine (SVM) for the damage identification of skeletal structures. The proposed method can be used to locate damage and identify damage patterns with the limited number of sensors. This paper is organized as follows. Firstly, the cross correlation function amplitude and support vector machine are introduced in Sections 2 and 3 , respectively. In Section 4, the damage detection method for civil engineering structures based on the CCFA and SVM is illustrated in detail. In addition, the simulation data of IASC-ASCE benchmark simulation model and a vibration experiment of truss structure are used to illustrate the feasibility of proposed method in Sections 5 and 6.

\section{Cross Correlation Function Amplitude (CCFA)}

The cross correlation function $R_{x, y}(T)$ of two stationary stochastic processes $x(t)$ and $y(t)$ with a time lag $T$ is defined as

$$
R_{x, y}(T)=E[x(t) y(t+T)],
$$

where $E[\cdot]$ is the expectation of the stochastic variable.

The equation of motion for $N$ degree-of-freedom (DOF) structure with classical damping is

$$
\mathbf{M} \ddot{\mathbf{X}}(t)+\mathbf{C} \dot{\mathbf{X}}(t)+\mathbf{K X}(t)=\mathbf{F}(t),
$$

where $\mathbf{X}(t)$ is the $N$-dimensional displacement vector, $\mathbf{F}(t)$ is the excitation vector, and $\mathbf{M}, \mathbf{C}$, and $\mathbf{K}$ denote, respectively, the mass, damping, and stiffness matrices of the structure with the dimension of $N \times N$. The displacement response can be decomposed into the $N$ modal coordinates as

$$
\mathbf{X}(t)=\sum_{n=1}^{N} \Phi^{n} Y^{n}(t)
$$

in which $\Phi^{n}$ is the $n$th modal vector and $Y^{n}(t)$ is the corresponding modal coordinate. The $n$th modal response can be separately written as

$$
\mathbf{X}^{n}(t)=\Phi^{n} Y^{n}(t)
$$

By Duhamel's integral, the $n$th modal response at point $i$ due to excitation $f_{k}(t)$ at point $k$ is

$$
X_{i}^{n}(t)=\phi_{i}^{n} \phi_{k}^{n} \int_{-\infty}^{t} f_{k}(\tau) g^{n}(t-\tau) d \tau
$$

where

$$
g^{n}(t-\tau)=\frac{\sin \omega_{D n}(t-\tau) \exp \left[-\xi_{n} \omega_{n}(t-\tau)\right]}{m_{n} \omega_{D n}}
$$

$m_{n}, \xi_{n}$, and $\omega_{n}$ are the modal mass, modal damping ratio, and natural frequency of the $n$th mode, respectively; and $\omega_{D n}=\omega_{n} \sqrt{1-\xi_{n}^{2}}$ is the damped natural frequency of the $n$th mode. For ambient loading, the exciting points are numerous, and $X_{i}^{n}(t)$ is the accumulation of responses caused by each exciting point as follows:

$$
X_{i}^{n}(t)=\phi_{i}^{n} \int_{-\infty}^{t} \sum \phi_{k}^{n} f_{k}(\tau) g^{n}(t-\tau) d \tau .
$$

Based on the natural excitation technique (NExT) [27], the cross correlation function between the $n$th modal responses at the $i$ th and $j$ th points can be written as

$$
\begin{gathered}
R_{x_{i}, x_{j}}^{n}(T)=\phi_{i}^{n} \phi_{j}^{n} \int_{-\infty}^{t} \int_{-\infty}^{t+T} g^{n}(t-\tau) g^{n}(t+T-\sigma) \\
\cdot \sum \sum \phi_{k}^{n} \phi_{k}^{n} E\left\{f_{k}(\tau) f_{k}(\sigma)\right\} d \sigma d \tau .
\end{gathered}
$$


If the ambient vibration source is a white noise random process, then

$$
E\left\{f_{k}(\tau) f_{k}(\sigma)\right\}=\alpha_{k} \delta(\tau-\sigma),
$$

where $2 \alpha_{k}$ is a constant representing the one-side autospectral density of the white noise and $\delta(t)$ is the Dirac delta function. Substituting (7) and (9) into (8), one can express the cross correlation function of the $n$th modal displacement responses at the $i$ th and $j$ th point as follows:

$$
R_{x_{i}, x_{j}}^{n}(T)=\frac{\phi_{i}^{n} G_{j}^{n}}{m_{n} \omega_{D n}} \exp \left(-\xi_{n} \omega_{n} T\right) \sin \left(\omega_{D n} T+\varphi_{n}\right),
$$

where $G_{j}^{n}$ is the coefficient depending on $n$th modal parameters, exciting points, and measured response at point $j$ and $\varphi_{n}$ is a phase dependent on the $n$th modal parameters. Since the structural responses under the white noise excitation are stationary stochastic processes, the cross correlation function of the $n$th modal velocity responses at the $i$ th and $j$ th points can be written as

$$
\begin{aligned}
& R_{\dot{x}_{i}, \dot{x}_{j}}^{n}(T)=-R_{x_{i}, x_{j}}^{n} \prime \prime(T)=\frac{\phi_{i}^{n} G_{j}^{n}}{m_{n} \omega_{D n}} \exp \left(-\xi_{n} \omega_{n} T\right) \\
& \cdot\left[\left(\omega_{D n}^{2}-\xi_{n}^{2} \omega_{n}^{2}\right) \sin \left(\omega_{D n} T+\varphi_{n}\right)\right. \\
& \left.+2 \xi_{n} \omega_{n} \omega_{D n} \cos \left(\omega_{D n} T+\varphi_{n}\right)\right] .
\end{aligned}
$$

Also, the cross correlation function of the $n$th modal acceleration responses at the $i$ th and $j$ th points can be expressed as

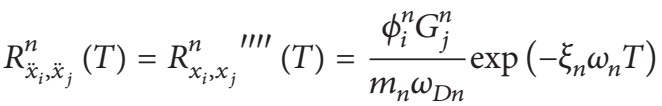

$$
\begin{aligned}
& \cdot\left[\left(\omega_{n}^{4}-6 \xi_{n}^{2} \omega_{n}^{2} \omega_{D n}^{2}+\omega_{D n}^{4}\right) \sin \left(\omega_{D n} T+\varphi_{n}\right)\right. \\
& \left.+\left(4 \xi_{n} \omega_{n} \omega_{D n}^{3}+4 \xi_{n}^{3} \omega_{n}^{3} \omega_{D n}\right) \cos \left(\omega_{D n} T+\varphi_{n}\right)\right] .
\end{aligned}
$$

Consequently, the cross correlation function amplitudes (CCFAs) of the $n$th modal displacement, velocity, and acceleration responses are

$$
\begin{aligned}
& \max {\left[R_{i, j}^{n}(T)\right]=\frac{G_{j}^{n}}{m_{n} \omega_{D n}} } \\
& \cdot \frac{a\left(a \omega_{D n}-b \xi_{n} \omega_{n}\right)+b\left(a \xi_{n} \omega_{n}+b \omega_{D n}\right)}{\sqrt{\left(a \omega_{D n}-b \xi_{n} \omega_{n}\right)^{2}+\left(a \xi_{n} \omega_{n}+b \omega_{D n}\right)^{2}}} \\
& \cdot \exp \left(-\xi_{n} \omega_{n} T^{*}\right) \phi_{i}^{n},
\end{aligned}
$$

where

$$
T^{*}=\arcsin \frac{a\left(a \omega_{D n}-b \xi_{n} \omega_{n}\right)}{\sqrt{\left(a \omega_{D n}-b \xi_{n} \omega_{n}\right)^{2}+\left(a \xi_{n} \omega_{n}+b \omega_{D n}\right)^{2}}} .
$$

The values of $a$ and $b$ have been listed in Table 1 .
TABLE 1: Values of $a$ and $b$ for the CCFA of $n$th modal displacement, velocity, and acceleration responses.

\begin{tabular}{lcc}
\hline$R_{i, j}^{n}(T)$ & $a$ & $b$ \\
\hline$R_{x_{i}, x_{j}}^{n}(T)$ & 1 & 0 \\
$R_{\dot{x}_{i}, \dot{x}_{j}}^{n}(T)$ & $\omega_{D n}^{2}-\xi_{n}^{2} \omega_{n}^{2}$ & $2 \xi_{n} \omega_{n} \omega_{D n}$ \\
$R_{\ddot{x}_{i}, \dot{x}_{j}}^{n}(T)$ & $\omega_{n}^{4}-6 \xi_{n}^{2} \omega_{n}^{2} \omega_{D n}^{2}+\omega_{D n}^{4}$ & $4 \xi_{n} \omega_{n} \omega_{D n}^{3}+4 \xi_{n}^{3} \omega_{n}^{3} \omega_{D n}$ \\
\hline
\end{tabular}

Define intermediate variable $\kappa^{*}\left(\xi_{n}, \omega_{n}\right)$ as

$$
\begin{aligned}
& \kappa^{*}\left(\xi_{n}, \omega_{n}\right)=\frac{a\left(a \omega_{D n}-b \xi_{n} \omega_{n}\right)+b\left(a \xi_{n} \omega_{n}+b \omega_{D n}\right)}{\sqrt{\left(a \omega_{D n}-b \xi_{n} \omega_{n}\right)^{2}+\left(a \xi_{n} \omega_{n}+b \omega_{D n}\right)^{2}}} \\
& \cdot \exp \left(-\xi_{n} \omega_{n} T^{*}\right) .
\end{aligned}
$$

The point $j$ is taken as a reference point, and the CCFAs of the $n$th modal signals between point $j$ and other points are calculated with their CCFAs listed as a vector,

$$
\begin{aligned}
& \mathbf{V}_{\mathrm{CCFA}}=\left[\max \left(R_{1, j}^{n}\right) \max \left(R_{2, j}^{n}\right) \cdots \max \left(R_{N, j}^{n}\right)\right] \\
& =\frac{G_{j}^{n} \kappa^{*}\left(\xi_{n}, \omega_{n}\right)}{m_{n} \omega_{D n}}\left[\begin{array}{llll}
\phi_{1} & \phi_{2} & \cdots & \phi_{N}
\end{array}\right] .
\end{aligned}
$$

$\mathbf{V}_{\text {CCFA }}$, which can be calculated from the displacement, velocity, or acceleration response, is closely related to the $n$th modal shape. When damage occurs, the modal shape close to the damaged location will be changed, and the damage feature $\mathbf{D}_{\text {CCFA }}$ is defined as the difference in $\mathbf{V}_{\text {CCFA }}$ between the intact and current conditions as

$$
\mathbf{D}_{\mathrm{CCFA}}=\mathbf{V}_{\mathrm{CCFA}}^{\text {intact }}-\mathbf{V}_{\mathrm{CCFA}}^{\text {current }},
$$

where the superscripts "intact" and "current" stand for $\mathbf{V}_{\text {CCFA }}$ at the intact and current conditions, respectively.

\section{Support Vector Machine (SVM)}

The support vector machine is a recently developed statistical learning algorithm based on the structural risk minimization (SRM) principle. The basic SVM deals with binary classification problems. The main idea of the SVM is to transform the data to a higher dimensional features space and to find the optimal hyperplane in the space that maximizes the margin between the two classes as shown in Figure 1. Consider a set of training data $\left\{\left(x_{i}, y_{i}\right), i=1,2, \ldots, l\right\}, x_{i}$ is the vector of input variables, and $y_{i}$ is the target vector corresponding to $\{-1,1\}$ in binary classification problem.

For the case of linear data, the separating hyperplane can be defined by

$$
f(x)=\mathbf{w}^{T} \mathbf{x}+b=0,
$$

where $f(x)$ is the separating hyperplane and the vectors $\mathbf{w}$ and $b$ are the weight factors defining the position of the separating hyperplane. The decision function is given by (19), 


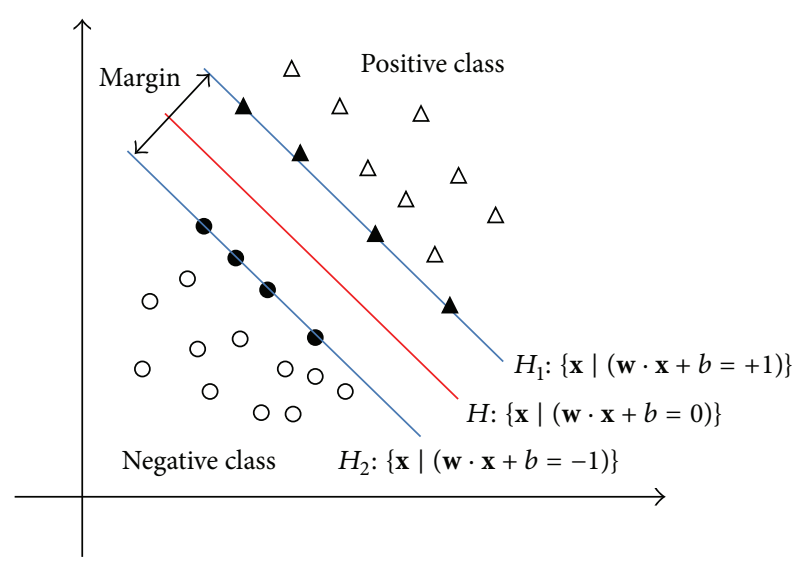

FIGURE 1: The diagram of SVM.

with which the input data are classified into either positive or negative class

$$
\tilde{f}(x)=\operatorname{sign}[f(x)] .
$$

Taking into account the noise with slack variables $\zeta_{i}$ and the error penalty $C$, the optimal hyperplane separating the data can be obtained by minimizing $\|\mathbf{w}\|$, leading to the following constrained optimization problem:

$$
\begin{aligned}
\min _{\omega, b, \zeta_{i}} & \frac{1}{2} \mathbf{w}^{T} \mathbf{w}+C \sum_{i=1}^{l} \zeta_{i} \\
\text { subject to } & y_{i}\left(\mathbf{w}^{T} \cdot x_{i}+b\right) \geq 1-\zeta_{i} \\
& \zeta_{i} \geq \mathbf{0}, \quad i=1, \ldots, l .
\end{aligned}
$$

Introducing the Lagrangian multiplier $\alpha_{i} \geq 0$, the above problem can be transformed to the following form:

$$
\begin{array}{ll}
\operatorname{maximize} & L(\mathbf{w}, b, \alpha)=\sum_{i=1}^{l} \alpha_{i}-\frac{1}{2} \sum_{i, j=0}^{l} \alpha_{i} \alpha_{j} y_{i} y_{j} x_{i} \cdot x_{j} \\
\text { subject to } & \alpha_{i} \geq 0 \\
& i=1, \ldots, M \\
& \sum_{i=1}^{M} \alpha_{i} y_{i}=0 .
\end{array}
$$

The linear SVM algorithm derived above can be easily extended to the nonlinear regression analysis by kernel methods. Using the nonlinear vector function $\Psi(\mathbf{x})=$ $\left(\psi_{1}(x), \ldots, \psi_{M}(x)\right)$ to map the $n$-dimensional input vector $\mathbf{x}$ onto the $l$-dimensional features space, the linear decision function in dual form is given by

$$
\tilde{f}(x)=\operatorname{sign}\left(\sum_{i, j=1}^{M} \alpha_{i} y_{i}\left(\Psi^{T}\left(x_{i}\right) \cdot \Psi\left(x_{j}\right)\right)+b\right) .
$$

The kernel function $K\left(x_{i}, x_{j}\right)=\left(\Psi^{T}\left(x_{i}\right) \cdot \Psi\left(x_{j}\right)\right)$ is applied to simplifying of the calculation, by which, the learning in the
TABLE 2: The commonly used kernel functions.

\begin{tabular}{lc}
\hline Linear & $K\left(x_{i}, x_{j}\right)=x_{i} \cdot x_{j}$ \\
Polynomial & $K\left(x_{i}, x_{j}\right)=\left(x_{i} \cdot x_{j}+c\right)^{d}$ \\
Radial Basis Function (RBF) & $K\left(x_{i}, x_{j}\right)=\exp \left(\gamma\left\|x_{i}-x_{j}\right\|^{2}\right)$ \\
Sigmoidal kernel function (SKF) & $K\left(x_{i}, x_{j}\right)=\tanh \left(\gamma\left\|x_{i}-x_{j}\right\|^{2}\right)$ \\
\hline
\end{tabular}

features space does not require explicit evaluation of $\Psi$ and the decision function will be

$$
\widetilde{f}(x)=\operatorname{sign}\left(\sum_{i, j=1}^{M} \alpha_{i} y_{i} K\left(x_{i}, x_{j}\right)+b\right) .
$$

Any function that satisfies Mercer's theorem can be used as a kernel function. The selection of kernel function is important to SVM, because the kernel function decides the future spaces that the samples will be mapped into. The commonly used kernel functions include the linear, polynomial, Gaussian Radial Basis Function (RBF), and sigmoidal kernel function (SKF), as listed in Table 2.

The fundamental capability of the SVM discussed above deals with binary classification. In practice, however, the method can be extended to solve multiclassification problems as well. One typical multiclassification method is called the one-against-one (OAO) algorithm $[15,28]$. This method uses $k(k-1) / 2$ binary classifiers to identify $k$ classes. Each training data piece is divided into two classes. For training data from the $i$ th and the $j$ th classes, the binary classification problem is shown as (24). If $x$ is in the $i$ th class, then the vote for the $i$ th class is added by one; otherwise, $j$ th is increased by one. When classifying test data, all of the SVMs perform classification and the test data is identified as the class with the largest number of votes. The schematic diagram of OAO algorithm is shown in Figure 2. Consider

$$
\begin{array}{ll}
\operatorname{minimize} & \frac{1}{2}\left\|\mathbf{w}^{i j}\right\|^{2}+C \sum_{t} \zeta_{t}^{i j}\left(\mathbf{w}^{i j}\right)^{T}, \\
\text { subject to } & \left(\mathbf{w}^{i j}\right)^{T} \phi\left(\mathbf{x}_{t}\right)+b^{i j} \leq 1-\zeta_{t}^{i j} \quad \text { if } y_{t}=i, \\
& \left(\mathbf{w}^{i j}\right)^{T} \phi\left(\mathbf{x}_{t}\right)+b^{i j} \leq-1+\zeta_{t}^{i j} \quad \text { if } y_{t}=j, \\
\zeta_{t}^{i j} \geq 0, \quad j=1, \ldots, l .
\end{array}
$$

\section{Damage Detection Based on CCFA and SVM}

In this paper, $\mathbf{D}_{\mathrm{CCFA}}$ is taken as the damage features for the input data vector, and the damage pattern can be classified by the multiclass SVM. There are two main advantages with the proposed data features. First, as revealed by (16), $\mathbf{V}_{\text {CCFA }}$ is a stable variable closely related to the structural modal shapes. Therefore, the accuracy of the method can hardly be interfered by external factors such as ambient excitation. In addition, because the relative change in the structural modal shape is sensitive to the damage, the difference of $\mathbf{V}_{\text {CCFA }}$ between the intact and current conditions is sensitive to minor damage with small stiffness changes. 


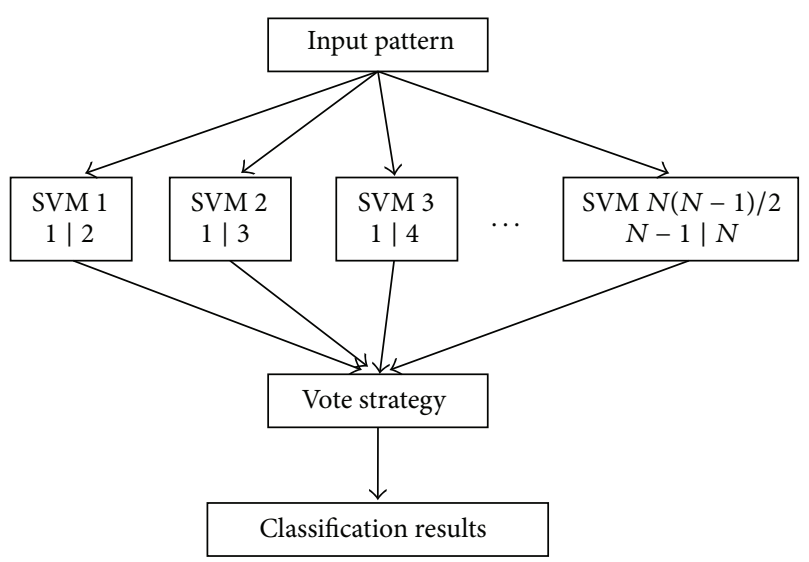

FIGURE 2: Schematic of OAO algorithm.

The procedure of the proposed method, as shown in Figure 3, can be summarized as follows.
(1) Assume that there are $M$ ordinary types of circumstances with the label of $1, \ldots, M$, respectively, in which 1 represents the intact condition and $2, \ldots, M$, respectively, stand for different damage patterns. Sample $N^{*}$ times of the dynamic response for each circumstance from $L$ sites of measurement, and take $N^{*} \times M \times L$ signals as samples.

(2) Extract the $n$th approximate modal response via some signal processing technologies such as WPT and EMD, by which, the dynamic responses can be decomposed, and, then, the approximate modal responses can be acquired by reconstructing the decomposed signals containing modal frequencies [29-31]. Then choose a special site, which is a node of mode insensitive to the damage, as the reference point for calculating $\mathbf{V}_{\text {CCFA }}$ based on (16) with the dimension of $1 \times L$. Or two reference points can be selected to ensure that the performance of identification is not affected by the reference point if one reference is sensitive to the damage. Then $\mathbf{V}_{\text {CCFA }}$ can be expressed as

$$
\mathbf{V}_{\mathrm{CCFA}}=\left[\max \left(R_{1, i}^{n}\right) \max \left(R_{2, i}^{n}\right) \cdots \max \left(R_{N, i}^{n}\right) \max \left(R_{1, j}^{n}\right) \max \left(R_{2, j}^{n}\right) \cdots \max \left(R_{N, j}^{n}\right)\right]
$$

(3) Calculate the data features, which are acquired from the difference of $\mathbf{V}_{\text {CCFA }}$ between the intact and current conditions according to (17). Then, the training data are established with $N^{*} \times M$ samples.

(4) The data features serve as the training data for input to the SVM and the classifiers are trained.

(5) Collect the test signals of the structure, and calculate the test samples with the same process from step (1) to step (4) as input data to the trained SVM classifiers. Then the structure status can be identified by the output of the SVM classifiers.

\section{Numerical Verification}

The simulation data of phase I IASC-ASCE benchmark model [32] is used to verify the feasibility of the method proposed in this paper. The sketch of the analytical model and the sensor locations is shown in Figure 4. In this paper, the symmetrical structure is simulated by a 12-DOF model, with each floor represented by 3 DOFs, including two translations and one rotation. The Gaussian white noise acceleration is loaded at all stories which can be represented as ambient excitation. The damage patterns are listed in Table 3 , in which pattern 0 is the intact, undamaged one, patterns 1 and 2 represent major damage patterns, and patterns 3 to 5 are minor damage patterns. Table 4 lists the percentage loss in the horizontal story stiffness of the damaged 12-DOF model for each pattern. As can be seen, patterns 1 and 2 represent major damage. The inclusion of patterns 3-5 is to test the ability of the proposed approach in detecting minor damage. In addition, pattern 3 is similar to pattern 4 , while pattern 0 is similar to pattern 5 .
The acceleration responses are calculated by the MATLAB program for the phase I benchmark model. There are 40 samples for the undamaged pattern and 20 samples for each damage pattern, respectively. Firstly, the first four approximate modal responses are extracted by the WPT technique. Figure 5 shows the original signal and first four modal acceleration responses at point 1 , and the FFT spectrum of each signal is shown in Figure 6. From Figure 6, one observes that the amplitudes of the first two frequencies in the original response are, respectively, equal to those in each approximate modal response, which can confirm the validity of the first two modal signals extracted by WPT technique.

Then, point 1 is taken as the reference point, and the value of $\mathbf{V}_{\text {CCFA }}$ is calculated using (15) from the first approximate modal responses. Twenty values of $\mathbf{V}_{\text {CCFA }}$ of pattern 0 are taken as the intact condition, and other values of $\mathbf{V}_{\text {CCFA }}$, containing twenty samples of pattern 0 and other five damage patterns, are taken as the current condition. Note that the cross correlation is a normalized sequence ranged from 0 to 1 which is calculated by MATLAB software, and the detailed principle can be found in [33]. The data features are defined by the difference of CCFA between the current and intact conditions. So there are 120 samples of data features for six patterns, and each pattern has 20 samples. Half of the samples in each pattern are used as the training data, while the others are used as the test data of the SVM. The training label of 1-6 represents pattern 0 , pattern $1, \ldots$, pattern 5 , subsequently. The multiclass classification system for the fault diagnostics with the one-against-one SVM is then established.

In this paper, the commonly used kernel functions including polynomial, RBF, and SKF are used to verify the 




FIgURE 3: Overall procedure of proposed method.

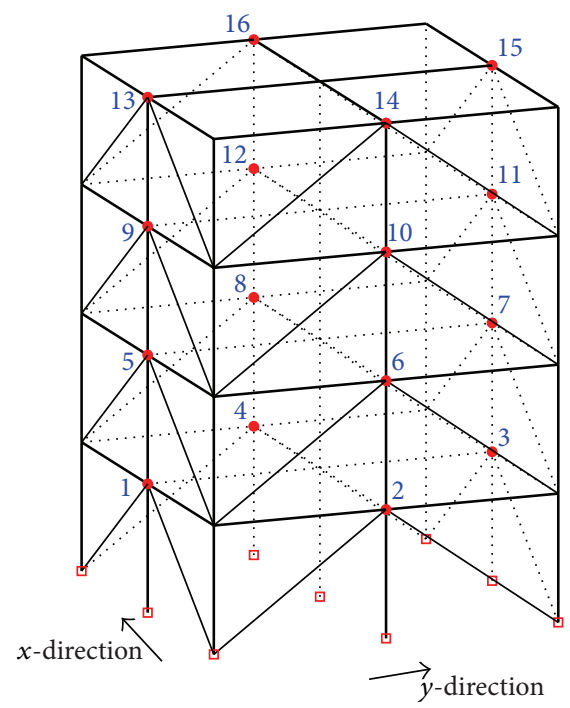

FIGURE 4: The sketch of the analytical model and the sensor locations.

superiority of CCFA based multiclass SVM. The SVM parameters play a crucial role in the performance of classification. There are two parameters for these kernel functions: $C$ and $\gamma$. In addition, the polynomial kernel function also has a parameter $d$, which means the degree of the polynomial. An improper choice of the parameters can cause the overfitting or underfitting problem. Therefore, the procedure of searching optimal parameters using training data is indispensable before model training. An exhaustive grid-search with exponentially growing $C$ and $\gamma$ is conducted to obtain the optimal choice of $C$ and $\gamma$ with the strategy of $v$-fold cross-validation [34]. In addition, the LIBSVM toolbox [35] is used for the procedure of the SVM.
TABLE 3: Damage patterns.

\begin{tabular}{ll}
\hline Damage pattern & Description \\
\hline Pattern 0 & Undamaged case \\
Pattern 1 & All braces of 1st story are broken \\
Pattern 2 & $\begin{array}{l}\text { All braces of 1st and 3rd stories are } \\
\text { broken }\end{array}$ \\
Pattern 3 & $\begin{array}{l}\text { brace on one side of 1st story is } \\
\text { broken } \\
\text { Pattern } 4\end{array}$ \\
Pattern 5 & $\begin{array}{l}\text { stories is broken } \\
\text { Area of } 1 \text { brace on one side of 1st } \\
\text { story is reduced to 2/3 }\end{array}$ \\
\hline
\end{tabular}
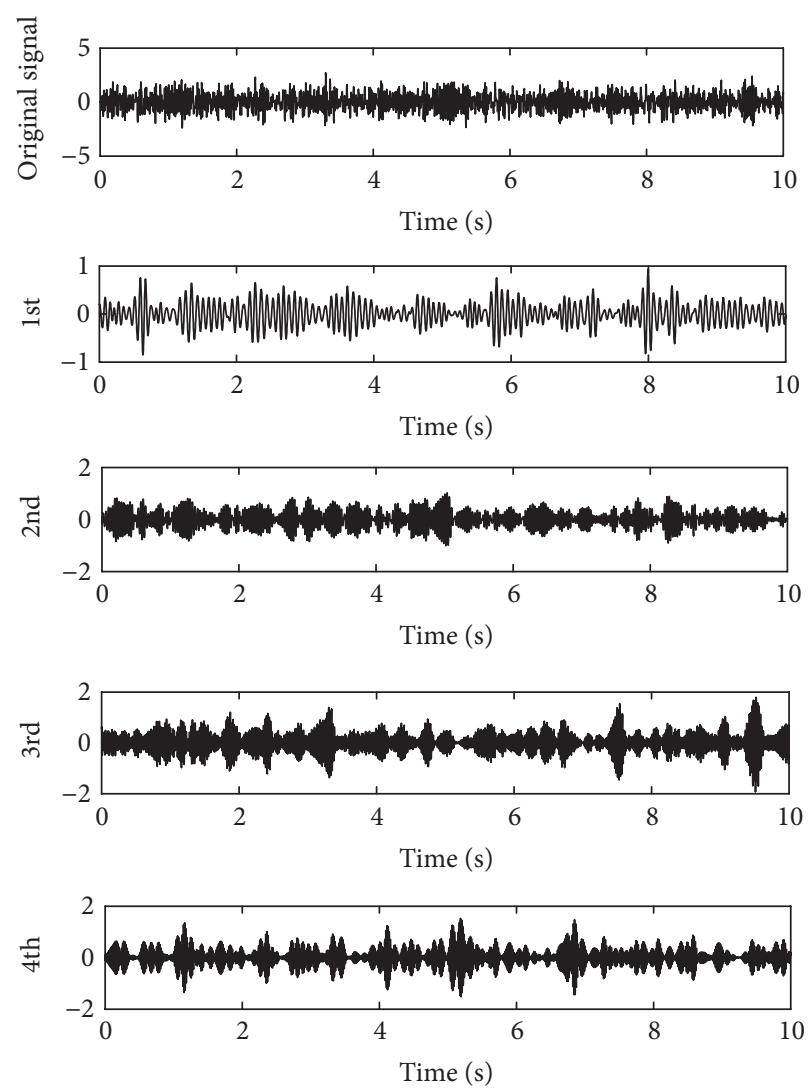

FIgURE 5: Original signal and first four approximate modal responses at point 1 .

Figure 7 shows the distribution of the $v$-fold cross validation $(\mathrm{CV})$ accuracy for different kernel functions. $C$ and $\gamma$ are ranged from $2^{-10}$ to $2^{10}$ exponentially, and $v$ is equal to 5. From Figure 7 the optimal combinations of $C$ and $\gamma$, which are those with the highest CV accuracy, are acquired.

In Table 5, the classification results of the testing samples are listed, in which $n / m$ means that there are $n$ valid identifications from $m$ samples. It can be seen that the SVM classifiers with the one-against-one algorithm can recognize the fault types effectively, especially with the kernel function of RBF. 
TABLE 4: Percentage losses in horizontal and rotational stiffness for each pattern.

\begin{tabular}{lcccccc}
\hline & Element & & \multicolumn{5}{c}{ Damage pattern } \\
Story & DOF & 1 & 2 & 3 & 4 & 5 \\
\hline 1 & $x$ & $45.24 \%$ & $45.24 \%$ & 0 & 0 & 0 \\
1 & $y$ & $71.03 \%$ & $71.03 \%$ & $17.76 \%$ & $17.76 \%$ & $5.92 \%$ \\
1 & $\theta$ & $64.96 \%$ & $64.96 \%$ & $9.87 \%$ & $9.87 \%$ & $0.88 \%$ \\
2 & $x$ & 0 & 0 & 0 & 0 & 0 \\
2 & $y$ & 0 & 0 & 0 & 0 & 0 \\
2 & $\theta$ & 0 & 0 & 0 & $11.31 \%$ & 0 \\
3 & $x$ & 0 & $45.24 \%$ & 0 & 0 & 0 \\
3 & $y$ & 0 & $71.03 \%$ & 0 & 0 & 0 \\
3 & $\theta$ & 0 & 0 & 0 & 0 & 0 \\
4 & $x$ & 0 & 0 & 0 & 0 & 0 \\
4 & $y$ & 0 & 0 & 0 & 0 & 0 \\
4 & $\theta$ & 0 & & 0 & & 0 \\
\hline
\end{tabular}

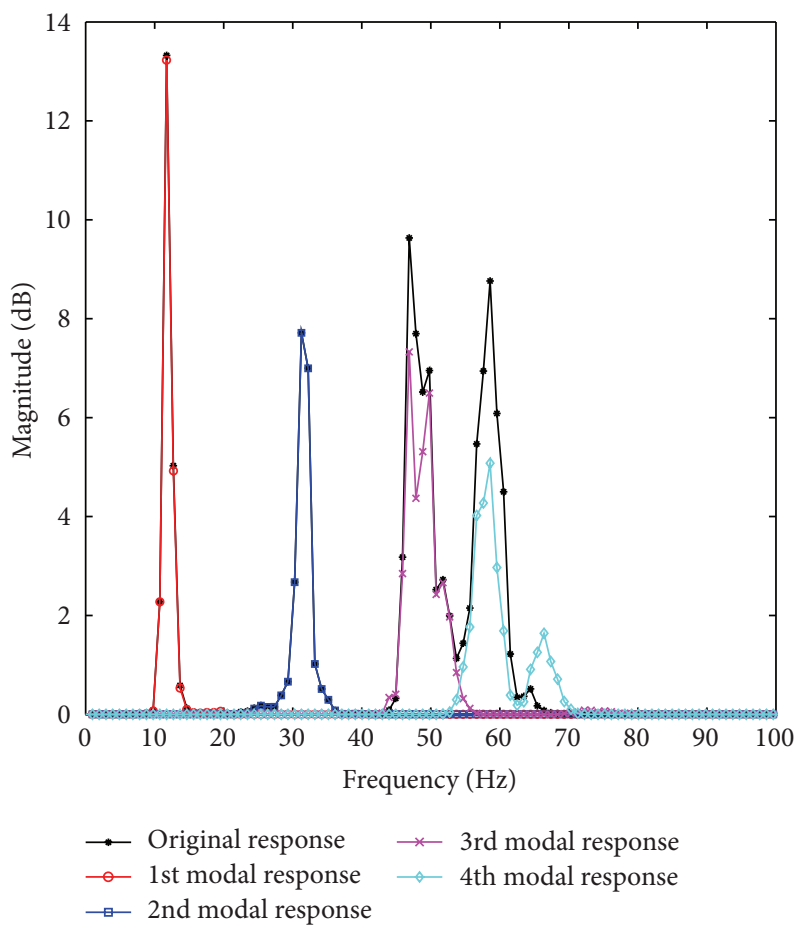

FIGURE 6: FFT spectrum of original signal and first 4 approximate modal responses.

The signals' energy is often used to measure the damage for civil engineering structures. The WPT energy spectrum and EMD energy spectrum are the classical damage features to depict the distributions of signals' energy [24, 36]. Meanwhile, the artificial neural network (ANN) is a classical intelligent algorithm which is used to pattern classification. To show the performance of the SVM classification scheme based on the CCFA features, a comparison is made between the proposed method, SVM classifications with other damage features including WPT energy spectrum and EMD energy spectrum, and ANN classification with CCFA features. The test accuracy rate of different method is shown in Figure 8, where WPT + SVM, EMD + SVM, and CCFA + SVM mean that the data features are extracted from the WPT spectrum, EMD spectrum, and CCFA, respectively. And CCFA + ANN means the ANN classification with CCFA features.

It can be seen that all methods can be used to identify major damage such as pattern 1 and pattern 2. However, the proposed method has the best classification performance for patterns 3,4, and 5, as a manifestation of minor damage of the benchmark model. Consequently, the superiority of the CCFA features based SVM has been demonstrated especially for the scenarios of minor damage with small stiffness changes.

The modal responses are extracted by signal process technologies. It is necessary to investigate the sensitivity of identification results to the veracity of extracting approximate modal response. To simulate the identification error, the approximate modal responses are modulated by adding noises with different levels. The identification results are shown in Figure 9, from which it can be seen that the average accuracy is still more than $90 \%$ when the approximate modal responses are mixed noise with a level of $20 \%$.

To study the effect of sensor noise on classification efficiency, a series of random noises are added to the responses of the structure with a level of 0 to $50 \%$ before the data features are extracted. Figure 10 shows the average accuracies of identification for patterns 0 to 3 with different classification method mentioned above for different levels of noises. The results show that a high level of accuracy can still be achieved even for the noise level up to $50 \%$ when the data features are extracted based on CCFA. Compared with data features extracted by other data features, the CCFA features based SVM method has been demonstrated to be better in noise robustness and is more appropriate for application to noisy environment. 
TABLE 5: The classification result of the testing samples.

\begin{tabular}{|c|c|c|c|c|c|c|}
\hline Kernel function & Pattern 0 & Pattern 1 & Pattern 2 & Pattern 3 & Pattern 4 & Pattern 5 \\
\hline Polynomial & $10 / 10$ & $10 / 10$ & $9 / 10$ & $10 / 10$ & $9 / 10$ & $9 / 10$ \\
\hline $\mathrm{KBF}$ & $10 / 10$ & $10 / 10$ & $10 / 10$ & $10 / 10$ & $10 / 10$ & $10 / 10$ \\
\hline SKF & $10 / 10$ & $10 / 10$ & $10 / 10$ & $10 / 10$ & $8 / 10$ & $9 / 10$ \\
\hline
\end{tabular}

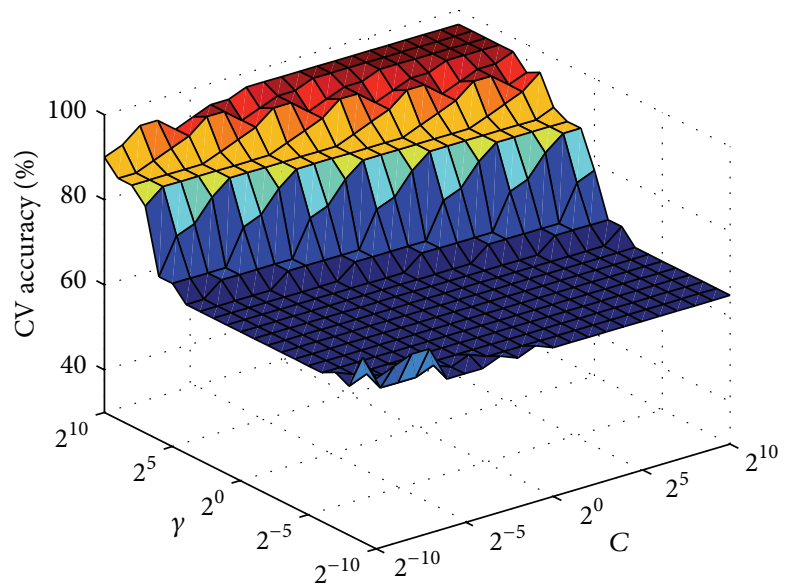

(a) Polynomial kernel function $(d=3)$. Optimal $C$ and $\gamma$ are $C=2^{-2}$, $\gamma=2^{10}$



(b) RBF kernel function. Optimal $C$ and $\gamma$ are $C=2^{-1}, \gamma=2^{8}$

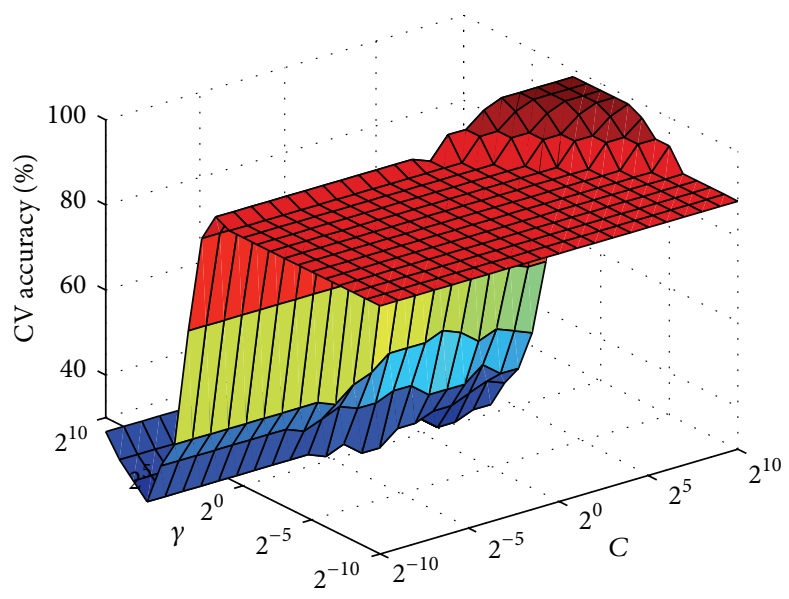

(c) SKF kernel function. Optimal $C$ and $\gamma$ are $C=2^{6}, \gamma=2^{2}$

FIGURE 7: CV accuracy with $C$ and $\gamma$ for polynomial, RBF, and SKF kernel functions, in the second loading condition.

\section{Experimental Verification}

A vibration experiment of truss structure is conducted to further verify the feasibility of proposed method with the limited number of sensors. The principal member of the structure is made by Q235 steel with the dimension of $L$ $30 \times 4$. The $300 \mathrm{~kg}$ weight stack is loaded on the top of the structure to guarantee the dynamical similarity. All members are connected by the bolts on the gusset plates. The experimental photo is shown in Figure 11. The loading devices are two JZK-20 vibration exciters, the matched signal generator and power amplifier. The dSPACE system is used as data acquisition system with the sample frequency of $500 \mathrm{~Hz}$.
The arbitrary voltage signal is generated by signal generator, then amplified by the power amplifier, and transferred to the vibration exciter to generate excitation forces on the top of truss structures.

The PZT (Pb-Based Lanthanum Doped Zirconate Titanates) sensors are attached on the surface of principal beams to collect the strain response of the structure as shown in Figure 12. All sensors are arrayed on one side of the truss model. The distribution of sensors location is shown in Figure 13, in which b1-b13 are the number of members and s- 1 to s- 6 are the number of PZT sensors.

The bolt looseness in gusset plate is one of the most common damage kinds for truss structures. In this paper, 


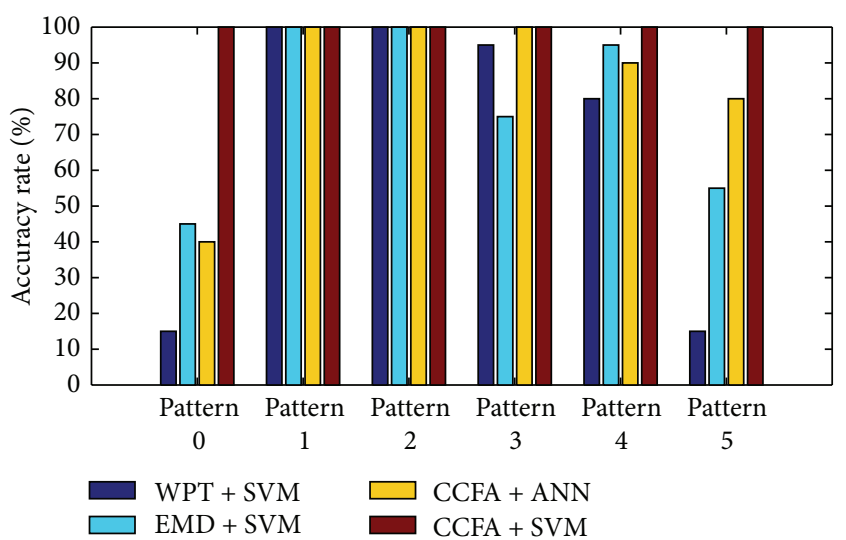

FIGURE 8: Test accuracy rate with different types of methods.

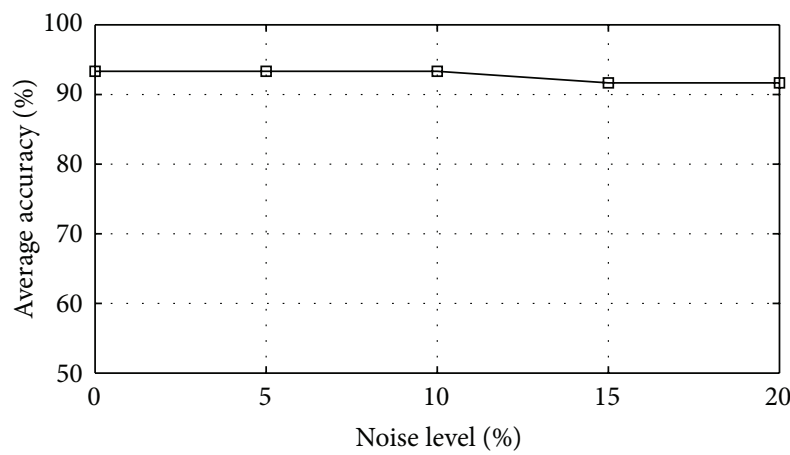

Figure 9: The identification results when the approximate modal responses are added with noises with different levels.

the bolts are loosened as the connecting damage in gusset plate. All the damage patterns are listed in Table 6. Note that no sensor is attached in b7, while the damage location in pattern 5 is far away from all sensors, which means that there is inadequate number of sensors distributed in the key locations. In addition, the damage patterns cannot be identified by natural frequencies.

The Gaussian white noise excitation with the frequency from 0 to $30 \mathrm{~Hz}$ and duration $20 \mathrm{~s}$ is loaded to the structure. 40 samples of PZT sensor responses in pattern 0 condition are acquired. 20 of them are used as initial samples, and the other are used as current undamaged samples. 20 samples in pattern 2-pattern 5 are collected as damaged samples.

The sensor s- 6 is taken as reference point, and then $\mathbf{V}_{\text {CCFA }}$ and $\mathbf{D}_{\mathrm{CCFA}}$ are calculated. Figure 14 shows the distribution of the $v$-fold cross-validation (CV) accuracy for different kernel functions, in which $C$ and $\gamma$ are ranged from $2^{-10}$ to $2^{10}$ exponentially, and $v$ is equal to 5 . Table 7 lists the classification accuracy of the truss structures for each damage pattern. It can be seen that the proposed method can identify the damage pattern in truss structure effectively with a limited number of sensors.

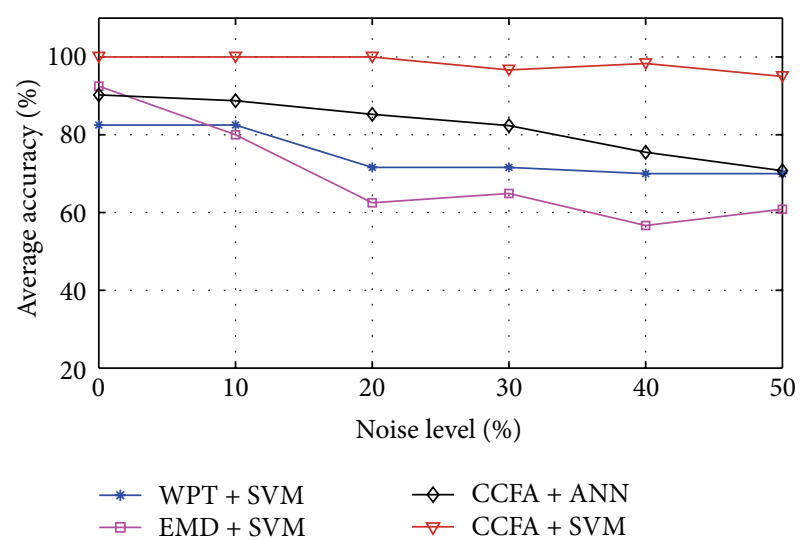

FIGURE 10: The average accuracies of identification with different level of noises.

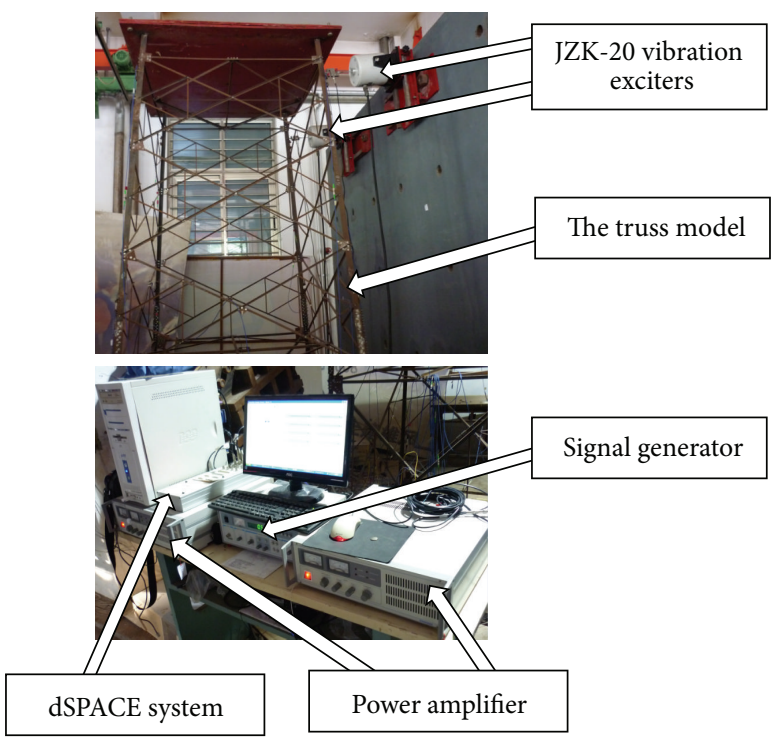

FIgURE 11: The experimental photo.



Figure 12: The PZT sensor. 
TABLE 6: The damage patterns of truss structure.

\begin{tabular}{lcc}
\hline Damage pattern & Description & Natural frequency \\
\hline Pattern 0 & Undamaged & $20.51 \mathrm{~Hz}$ \\
Pattern 1 & The bolts in the b5 connected b1, b3, and b7 are loosened & $16.6 \mathrm{~Hz}$ \\
Pattern 2 & The bolts in the joint connected b1, b3, and b7 are loosened & $16.6 \mathrm{~Hz}$ \\
Pattern 3 & The bolts in b1 connected with b3 are loosened & $17.58 \mathrm{~Hz}$ \\
Pattern 4 & The bolts in b7 connected with b1 and b3 are loosened & $20.51 \mathrm{~Hz}$ \\
Pattern 5 & A bottom column on the other side of structure is broken & $20.51 \mathrm{~Hz}$ \\
\hline
\end{tabular}

TABLE 7: The accuracy of damage pattern classification with different kernel function.

\begin{tabular}{lcccccc}
\hline Kernel function & Case 1 & Case 2 & Case 3 & Case 4 & Case 5 & Case 6 \\
\hline Polynomial & $100 \%$ & $80 \%$ & $100 \%$ & $80 \%$ & $70 \%$ & $100 \%$ \\
RBF & $100 \%$ & $80 \%$ & $100 \%$ & $90 \%$ & $90 \%$ & $100 \%$ \\
SKF & $100 \%$ & $80 \%$ & $100 \%$ & $80 \%$ & $80 \%$ & $100 \%$ \\
\hline
\end{tabular}

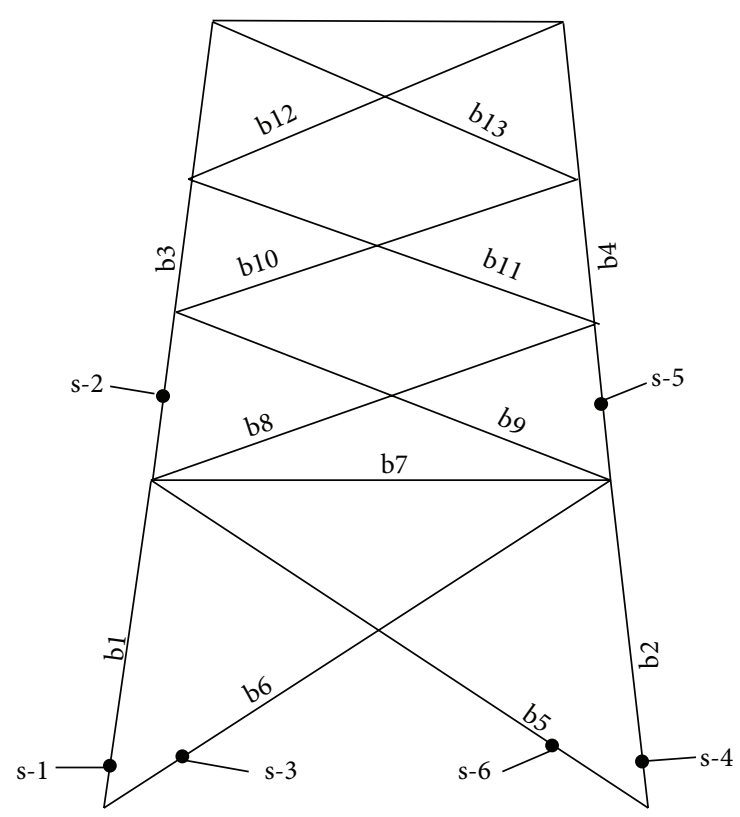

Figure 13: The distribution of PZT sensors.

\section{Concluding Remarks}

In this research, a damage detection approach integrating the cross correlation function amplitude (CCFA) with support vector machine (SVM) is proposed. The accuracy and stability of the SVM classification depend largely on the quality of input data features. The CCFA, closely related to the modal property of the structure, varies with the change in structural damage status. Therefore, the input data features can be extracted from the difference in CCFA between the current and intact conditions. This forms the basis for the proposed damage detection method that integrates the CCFA with SVM. The signal process technology such as the wavelet packet transform (WPT) or empirical mode decomposition (EMD) is used to extract single $n$th approximate modal response of the dynamic response, by which the data feature based on the CCFA is extracted. Normally, the 1st and 2nd mode signals are used in the detection. Next, the data features are used as input into the SVM for the classification of structural damage.

The proposed approach has been verified by the simulation data of the IASC-ASCE benchmark model and a vibration experiment of truss model, by which the properties of the proposed method, along with the advantages, can be outlined as follows:

(1) The CCFA features based SVM fault diagnosis approach shows excellent capability in damage classification and identification for the benchmark model and is suitable for the damage detection of engineering structures subjected to ambient excitation.

(2) The proposed approach can be used to identify minor damage for the benchmark model. It is concluded that the damage detection approach based on the integrated use of the CCFA and SVM is more reliable for identifying minor damage with small stiffness changes, in comparison with other approaches analyzed herein.

(3) The proposed method remains quite high in terms of accuracy for the benchmark model even if the noise level has been raised to $50 \%$. This is a demonstration that the proposed method has better noise robustness and is more appropriate for application in noisy environments.

(4) From the vibration experiment of truss model, it can be concluded that the proposed method is feasible for skeletal structures with a limited number of sensors. 


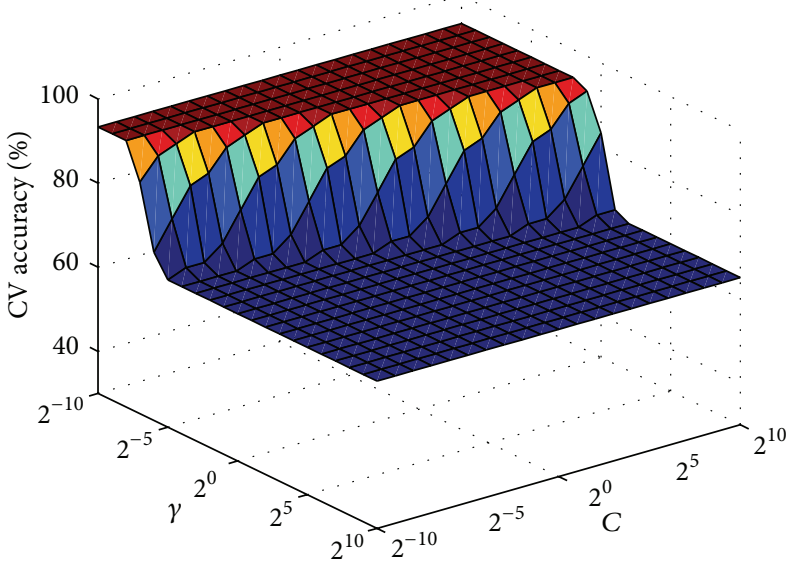

(a) Polynomial kernel function $(d=3)$. Optimal $C$ and $\gamma$ are $C=2^{-10}$, $\gamma=2^{8}$

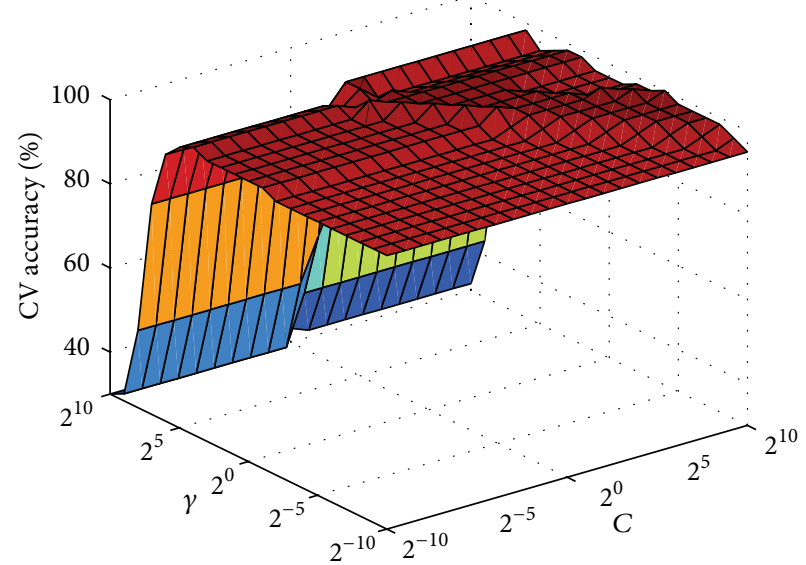

(b) RBF kernel function. Optimal $C$ and $\gamma$ are $C=2^{-1}, \gamma=2^{3}$

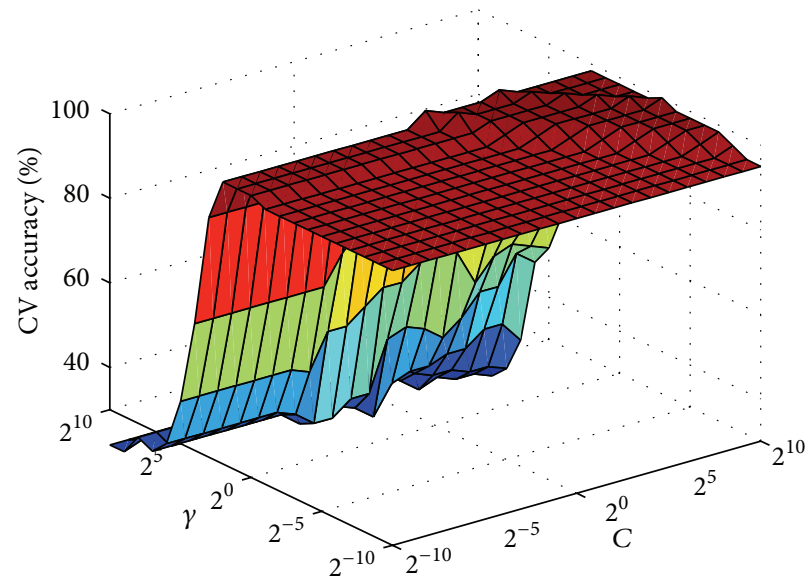

(c) SKF kernel function. Optimal $C$ and $\gamma$ are $C=2^{1}, \gamma=2^{2}$

FIgURE 14: The CV accuracy with $C$ and $\gamma$ for polynomial, RBF, and SKF kernel functions.

\section{Competing Interests}

The authors declare that they have no competing interests.

\section{Acknowledgments}

The authors would like to appreciate the support from National Natural Science Foundation of China (Grant no. 51421064) and 973 Program of China (no. 2015CB057704).

\section{References}

[1] Y. J. Yan, L. Cheng, Z. Y. Wu, and L. H. Yam, "Development in vibration-based structural damage detection technique," Mechanical Systems and Signal Processing, vol. 21, no. 5, pp. 2198-2211, 2007.

[2] Z. Yang, L. Wang, H. Wang, Y. Ding, and X. Dang, "Damage detection in composite structures using vibration response under stochastic excitation," Journal of Sound and Vibration, vol. 325, no. 4-5, pp. 755-768, 2009.

[3] J.-T. Kim, Y.-S. Ryu, H.-M. Cho, and N. Stubbs, "Damage identification in beam-type structures: frequency-based method vs mode-shape-based method," Engineering Structures, vol. 25, no. 1, pp. 57-67, 2003.

[4] K. Worden, G. Manson, and N. R. J. Fieller, "Damage detection using outlier analysis," Journal of Sound and Vibration, vol. 229, no. 3, pp. 647-667, 2000.

[5] C. Zang, M. I. Friswell, and M. Imregun, "Structural damage detection using independent component analysis," Structural Health Monitoring, vol. 3, no. 1, pp. 69-83, 2004.

[6] A. Hera and Z. Hou, "Application of wavelet approach for ASCE structural health monitoring benchmark studies," Journal of Engineering Mechanics, vol. 130, no. 1, pp. 96-104, 2004.

[7] D. Cantero and B. Basu, "Railway infrastructure damage detection using wavelet transformed acceleration response of traversing vehicle," Structural Control and Health Monitoring, vol. 22, no. 1, pp. 62-70, 2015.

[8] J.-P. Tang, D.-J. Chiou, C.-W. Chen et al., "RETRACTED: a case study of damage detection in benchmark buildings using a Hilbert-Huang Transform-based method," Journal of Vibration and Control, vol. 17, no. 4, pp. 623-636, 2011.

[9] J. N. Yang, Y. Lei, S. Lin, and N. Huang, "Hilbert-Huang based approach for structural damage detection," Journal of Engineering Mechanics, vol. 130, no. 1, pp. 85-95, 2004. 
[10] Z. Yang, Z. Yu, and H. Sun, "On the cross correlation function amplitude vector and its application to structural damage detection," Mechanical Systems and Signal Processing, vol. 21, no. 7, pp. 2918-2932, 2007.

[11] L. Wang, Z. Yang, and T. P. Waters, "Structural damage detection using cross correlation functions of vibration response," Journal of Sound and Vibration, vol. 329, no. 24, pp. 5070-5086, 2010.

[12] L. Wang and Z. Yang, "Effect of response type and excitation frequency range on the structural damage detection method using correlation functions of vibration responses," Journal of Sound and Vibration, vol. 332, no. 4, pp. 645-653, 2013.

[13] W. T. Yeung and J. W. Smith, "Damage detection in bridges using neural networks for pattern recognition of vibration signatures," Engineering Structures, vol. 27, no. 5, pp. 685-698, 2005.

[14] Y. Qian and A. Mita, "Acceleration-based damage indicators for building structures using neural network emulators," Structural Control and Health Monitoring, vol. 15, no. 6, pp. 901-920, 2008.

[15] A. Widodo and B.-S. Yang, "Support vector machine in machine condition monitoring and fault diagnosis," Mechanical Systems and Signal Processing, vol. 21, no. 6, pp. 2560-2574, 2007.

[16] H.-X. He and W.-M. Yan, "Structural damage detection with wavelet support vector machine: introduction and applications," Structural Control and Health Monitoring, vol. 14, no. 1, pp. 162-176, 2007.

[17] A. Widodo and B.-S. Yang, "Wavelet support vector machine for induction machine fault diagnosis based on transient current signal," Expert Systems with Applications, vol. 35, no. 1-2, pp. 307-316, 2008.

[18] Y. Q. Ni, X. G. Hua, K. Q. Fan, and J. M. Ko, “Correlating modal properties with temperature using long-term monitoring data and support vector machine technique," Engineering Structures, vol. 27, no. 12, pp. 1762-1773, 2005.

[19] K. Worden and A. J. Lane, "Damage identification using support vector machines," Smart Materials and Structures, vol. 10, no. 3 , pp. 540-547, 2001.

[20] C. K. Oh and H. Sohn, "Damage diagnosis under environmental and operational variations using unsupervised support vector machine," Journal of Sound and Vibration, vol. 325, no. 1-2, pp. 224-239, 2009.

[21] H. Song, L. Zhong, and B. Han, "Structural damage detection by integrating independent component analysis and support vector machine," International Journal of Systems Science, vol. 37, no. 13, pp. 961-967, 2006.

[22] Y. Yang, D. Yu, and J. Cheng, "A fault diagnosis approach for roller bearing based on IMF envelope spectrum and SVM," Measurement, vol. 40, no. 9-10, pp. 943-950, 2007.

[23] W. Sun, G. A. Yang, Q. Chen, A. Palazoglu, and K. Feng, "Fault diagnosis of rolling bearing based on wavelet transform and envelope spectrum correlation," Journal of Vibration and Control, vol. 19, no. 6, pp. 924-941, 2013.

[24] Q. Hu, Z. He, Z. Zhang, and Y. Zi, "Fault diagnosis of rotating machinery based on improved wavelet package transform and SVMs ensemble," Mechanical Systems and Signal Processing, vol. 21, no. 2, pp. 688-705, 2007.

[25] F. He and W. Shi, "WPT-SVMs based approach for fault detection of valves in reciprocating pumps," in Proceedings of the American Control Conference (ACC '02), vol. 6, pp. 4566-4570, IEEE, Anchorage, Alaska, USA, May 2002.

[26] A. Cury and C. Crémona, "Pattern recognition of structural behaviors based on learning algorithms and symbolic data concepts," Structural Control and Health Monitoring, vol. 19, no. 2, pp. 161-186, 2012.
[27] C. R. Farrar and G. H. James III, "System identification from ambient vibration measurements on a bridge," Journal of Sound and Vibration, vol. 205, no. 1, pp. 1-18, 1997.

[28] C.-W. Hsu and C.-J. Lin, "A comparison of methods for multiclass support vector machines," IEEE Transactions on Neural Networks, vol. 13, no. 2, pp. 415-425, 2002.

[29] R. R. Coifman and M. V. Wickerhauser, "Entropy-based algorithms for best basis selection," IEEE Transactions on Information Theory, vol. 38, no. 2, pp. 713-718, 1992.

[30] Z. Sun and C. C. Chang, "Structural damage assessment based on wavelet packet transform," Journal of Structural Engineering, vol. 128, no. 10, pp. 1354-1361, 2002.

[31] N. E. Huang, Z. Shen, S. R. Long et al., "The empirical mode decomposition and the Hilbert spectrum for nonlinear and non-stationary time series analysis," Proceedings of the Royal Society of London A: Mathematical, Physical and Engineering Sciences, vol. 454, no. 1971, pp. 903-995, 1998.

[32] E. A. Johnson, H. F. Lam, L. S. Katafygiotis, and J. L. Beck, "Phase I IASC-ASCE structural health monitoring benchmark problem using simulated data," Journal of Engineering Mechanics, vol. 130, no. 1, pp. 3-15, 2004.

[33] S. J. Orfanidis, Optimum Signal Processing. An Introduction, Prentice-Hall, Englewood Cliffs, NJ, USA, 2nd edition, 1996.

[34] C. W. Hsu, C. C. Chang, and C. J. Lin, "A practical guide to support vector classification," Tech. Rep., Department of Computer Science and Information Engineering, National Taiwan University, Taipei, Taiwan, 2003, http://www.csie.ntu .edu.tw/ cjlin/papers/guide/guide.pdf.

[35] C.-C. Chang and C.-J. Lin, "LIBSVM: a library for support vector machines," Tech. Rep., Department of Computer Science and Information Engineering, National Taiwan University, 2001, http://www.cs.cmu.edu/ pakyan/compbio/ references/Chang_LIBSVM_2001.pdf.

[36] J.-G. Han, W.-X. Ren, and Z.-S. Sun, "Wavelet packet based damage identification of beam structures," International Journal of Solids and Structures, vol. 42, no. 26, pp. 6610-6627, 2005. 


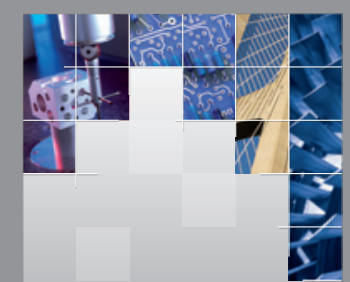

\section{Enfincering}
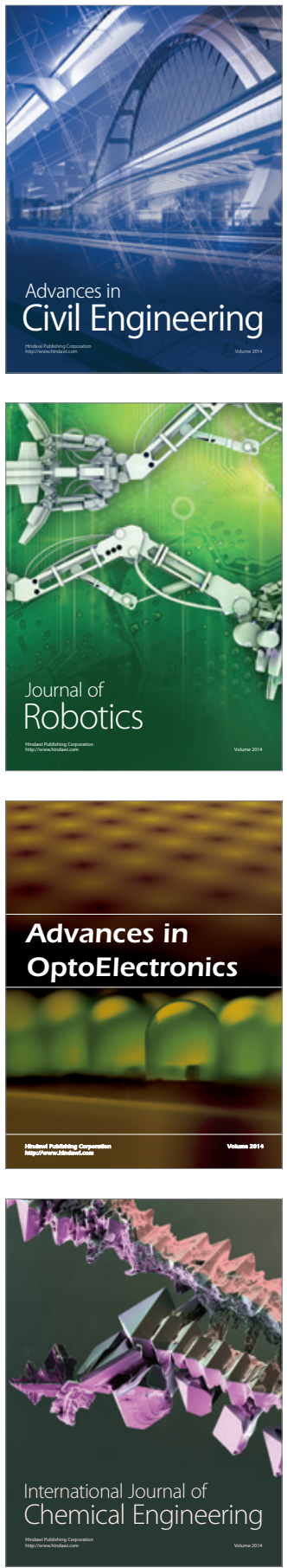

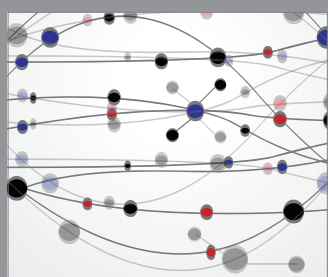

The Scientific World Journal

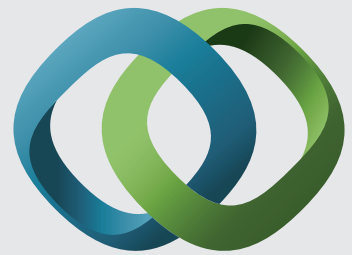

\section{Hindawi}

Submit your manuscripts at

http://www.hindawi.com

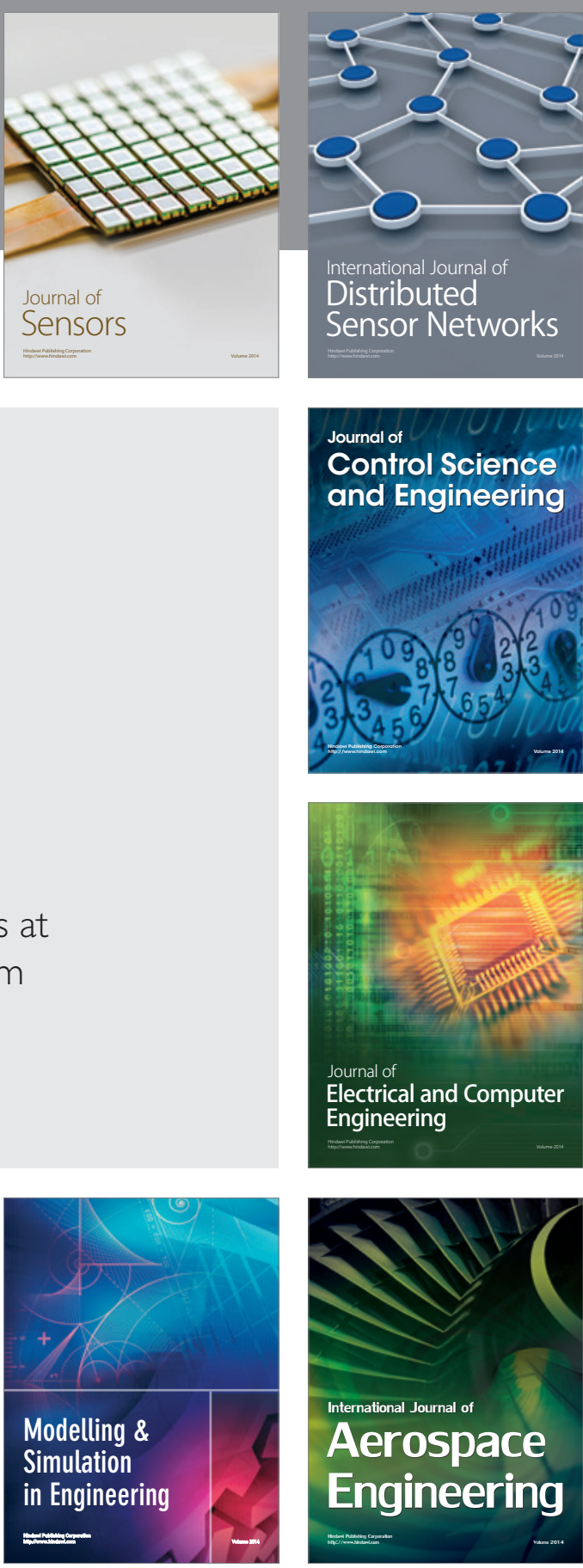

International Journal of

Distributed

Sensor Networks

Journal of

Control Science

and Engineering
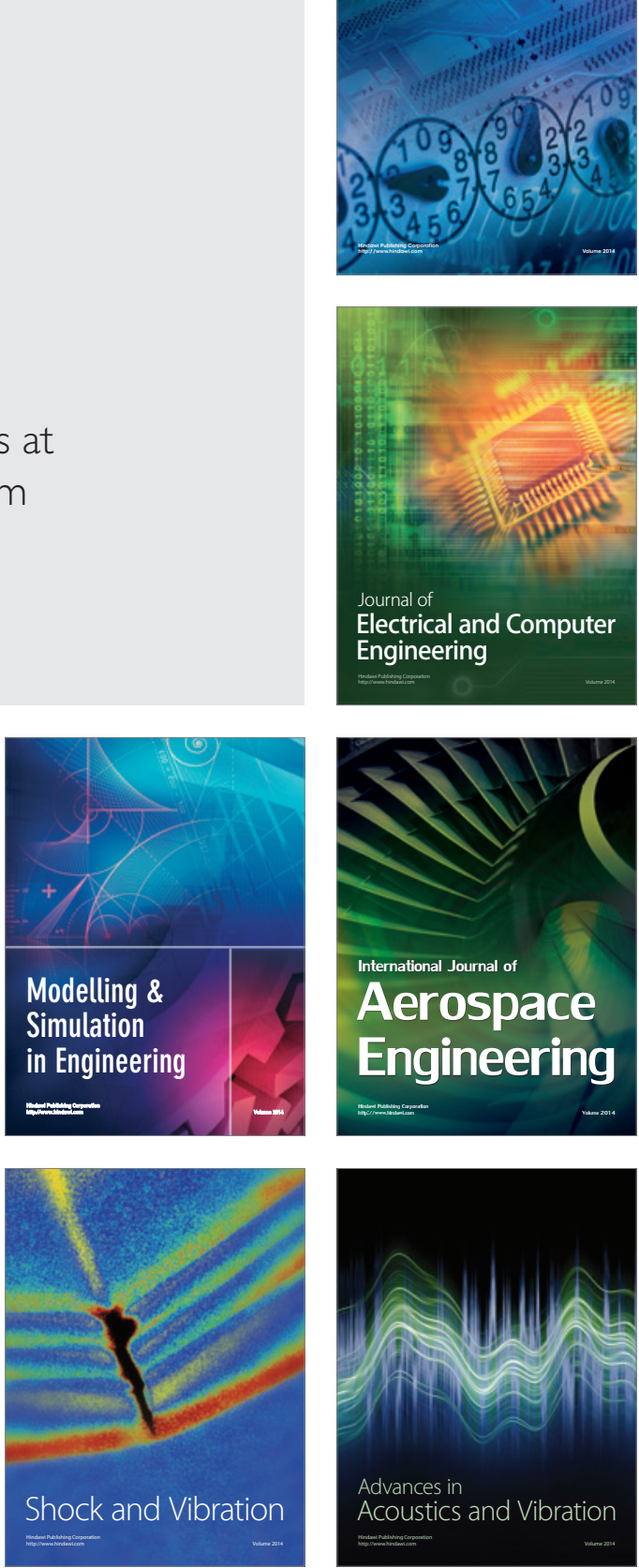